\title{
Palliative Care of Malignant Fibrous Histiocytoma of Spine with Cord Compression and Multiple Bone Metastases Treated by Multidisciplinary Therapy: Case Report
}

\author{
Ivan Sekiguchi $^{a} \quad$ Naoki Takeda ${ }^{b}$ Naoki Ishida ${ }^{a}$ \\ aDepartment of Orthopedic Surgery, Hokuto Medical Corporation Hokuto Hospital, \\ Obihiro, Japan; bepartment of Rehabilitation, Sapporo Orthopedic Cardiovascular \\ Hospital, Sapporo, Japan
}

\section{Keywords}

Malignant fibrous histiocytoma of spine $\cdot$ Spine sarcoma

\begin{abstract}
Malignant fibrous histiocytoma (MFH) of the spine is rare, with only a few dozen cases reported in the literature. A 60-year-old male was referred to us with symptoms of thoracic myelopathy. A solid tumor in the Th8 right costovertebral junction invading the spinal canal and compressing the spinal cord, and multiple bony metastases were discovered. Biopsy confirmed MFH. The thoracic spine tumor showed good response to irradiation followed by embolization and partial resection. The patient was followed until his death 22 months later. A good quality of life was sustained for more than 18 months. Despite a poor prognosis and an aggressive course of MFH of the spine, a good quality of life could be sustained for more than a year with palliative interventions.

\section{Introduction}

Malignant fibrous histiocytoma (MFH) was first described in 1964 [1]. MFH of the spine was first reported in 1976 [2]. MFH is diagnosed in 1/4 of patients in sarcoma clinical trials and mostly occurs in extremities and soft tissues of the trunk [3].

MFH of the spine is rare and has a much worse prognosis [4]. The diagnosis of MFH requires immunohistochemistry tests revealing no line of differentiation and is considered a 

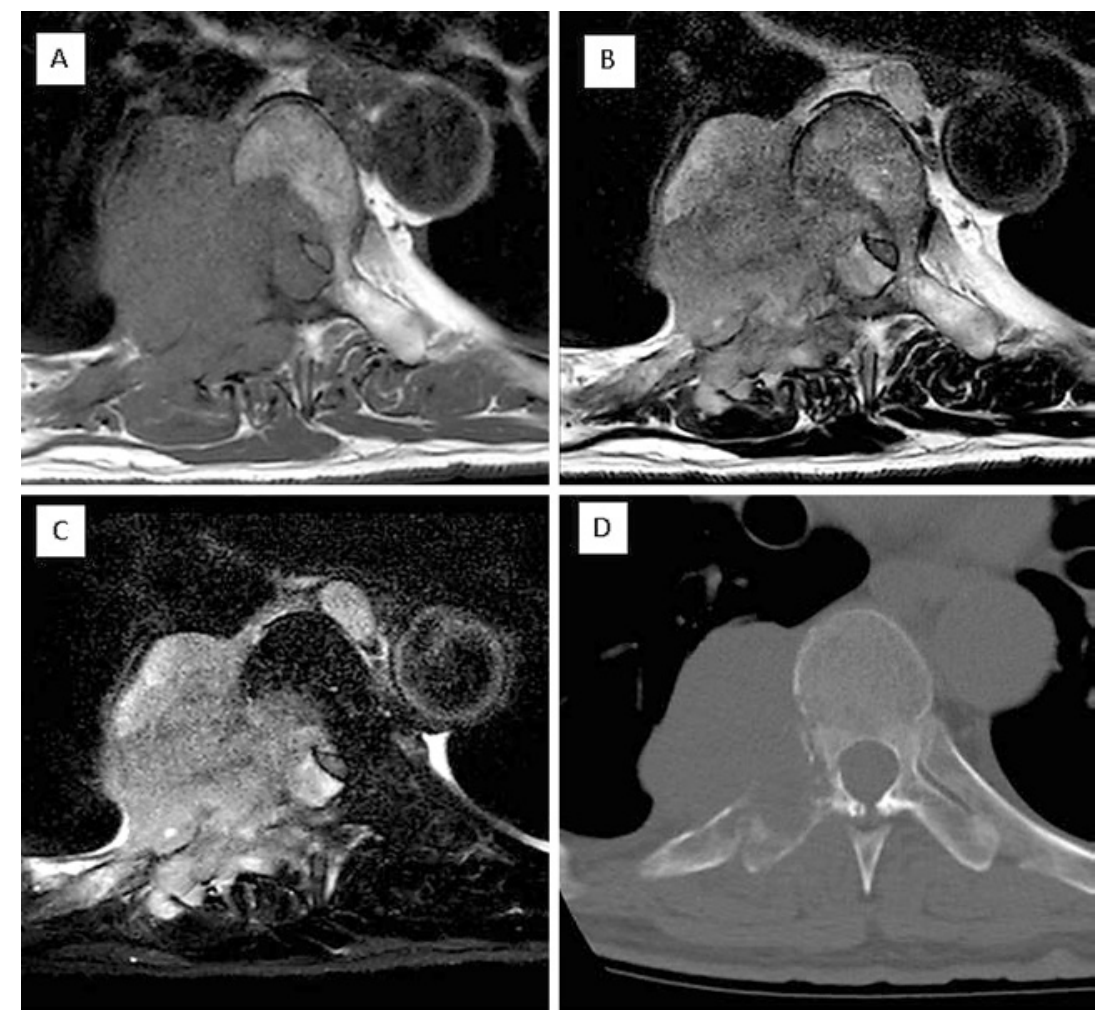

Fig. 1. Axial view of Th8 right rib junction tumor. A MRI T1. B MRI T2. C MRI fat suppression. D CT showing bone destruction without periosteal reaction or new bone formation.

diagnosis of exclusion [5]. We report a case of MFH in the thoracic spine, which presented with spinal cord compression and multiple bone metastases and was managed with good results with radiation, embolization, and palliative surgery.

\section{Case Report}

A 60-year-old man with a history of brain infarction and diabetes mellitus was referred to us with a suspicion of spinal cord injury after a fall. At the time of admission he had Frankel $\mathrm{C}$ grade paraparesis and urinary retention. Magnetic resonance imaging (MRI), computer tomography (CT), and positron emission tomography scan disclosed a tumor measuring $7 \mathrm{~cm}$ in the largest dimension at the Th8 right costal junction with bone destruction, and spinal canal invasion (Fig. 1), L2 vertebral body, right 10th rib and right femur metastasis were also found. Informed consent for treatment was obtained from the patient and his family. Emergency irradiation to the Th8 tumor of $8 \mathrm{~Gy} / 1 \mathrm{Fr}$ was administered and Th7, 8, 9 segmental artery embolization was performed. The patient was operated on the 6th day from admission after his clopidogrel withdrawal period. Percutaneous biopsy from L2 vertebral body was performed followed by Th7, 8, 9 laminectomy and removal of the tumor mass invading the spinal canal. There were no adhesions between tumor and nerve tissue. Intraoperative bleeding was negligible.

Pathology of the Th8 tumor revealed pleomorphic cells with dispersed spindle cells, Mib-1 index >20\%, p53 diffusely positive (Fig. 2). A small number of cells was S-100 positive. 


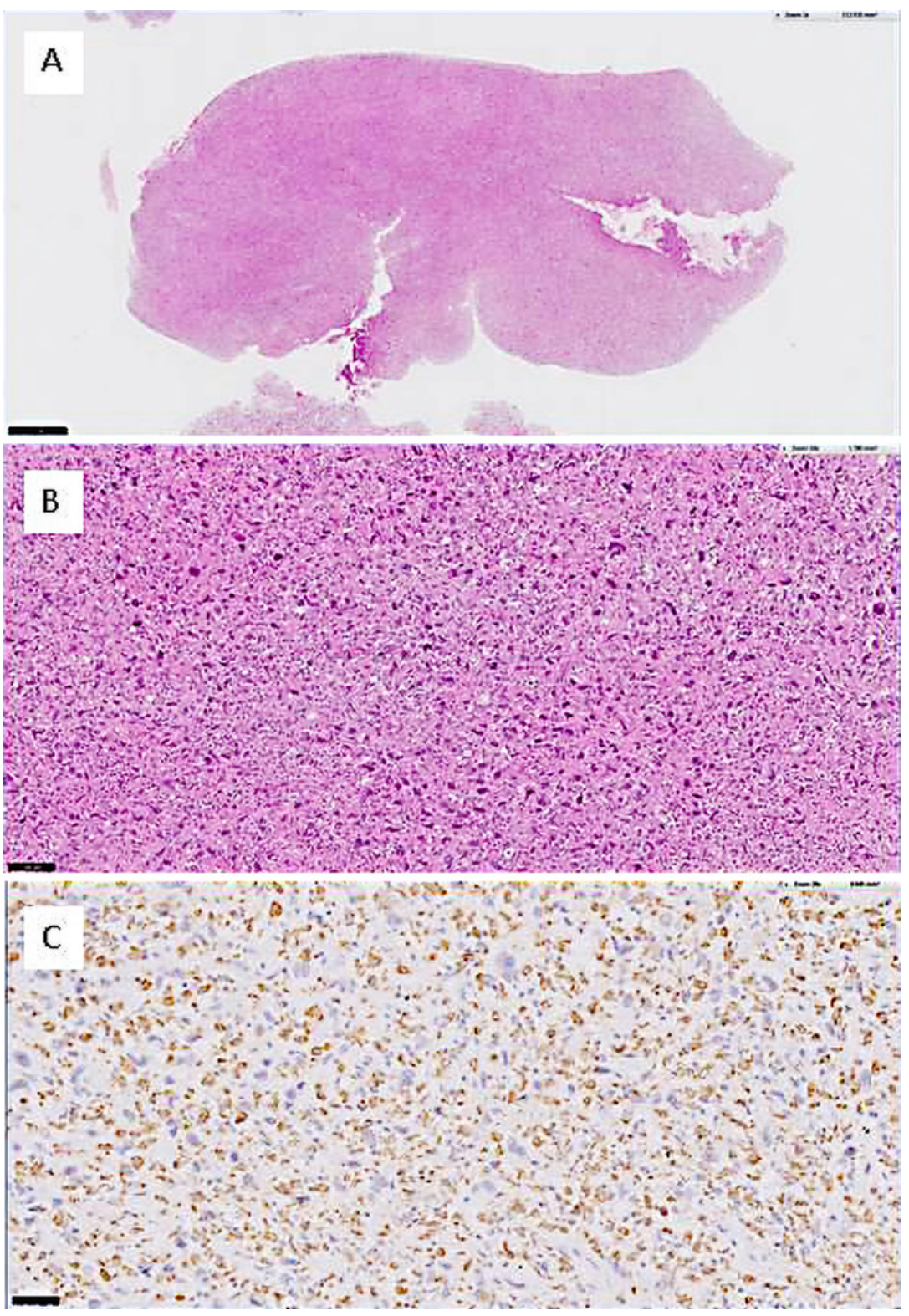

Fig. 2. A H\&E stain. $\times 20$ magnification. B H\&E stain. $\times 200$ magnification showing whorls of undifferentiated cells with atypical nuclei. C CD68 immunohistochemistry. $\times 400$ magnification showing diffusely positively stained cells.

Additional immunohistochemistry revealed AE1/AE3(-), aSMA(-), STAT6(-), CD34(-), $\operatorname{PgR}(-)$, NapsinA(-), TTF-1(-), CD68(+), cKit(-), GFAP(-).

L2 pathology was S-100(-) and showed less polymorphism.

Next, percutaneous instrumentation from L1 to L3 was performed for impending pathological fracture of L2 after $8 \mathrm{~Gy} / 1 \mathrm{Fr}$ irradiation to L2 body metastasis. Intramedullary nailing was performed for impending pathological fracture of the right femur after 24 Gy/4 Fr irradiation.

The postoperative course was benign. Paraparesis gradually improved to Frankel D within 3 months. The patient regained the ability to ambulate with a walker and normal urinary function. 


\section{Case Reports in \\ Oncology}

\begin{tabular}{l|l}
\hline Case Rep Oncol 2020;13:12-16 \\
\hline DOI: 10.1159/000504931 & $\begin{array}{l}\text { @ 2020 The Author(s). Published by S. Karger AG, Basel } \\
\text { www.karger.com/cro }\end{array}$ \\
\hline
\end{tabular}

Sekiguchi et al.: MFH of Spine Treated by Multidisciplinary Therapy
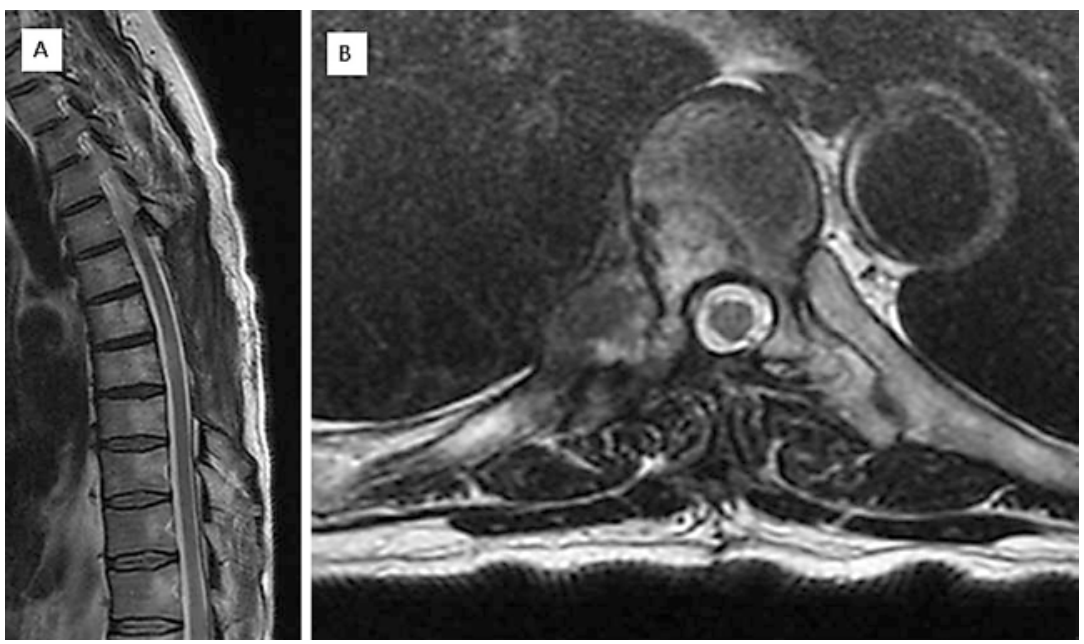

Fig. 3. Th8 tumor MRI T2 sagittal (A) and axial (B) slice at 1 year postoperatively showing significant tumor shrinking after irradiation, embolization, and partial resection.

The right 10th rib metastasis was controlled with a course of $66 \mathrm{~Gy} / 33 \mathrm{Fr}$ resulting in tumor shrinking.

Th8 tumor at this point shrank to $2 \mathrm{~cm}$ on MRI (Fig. 3). L2 and right femur metastases showed no progression. The patient was discharged home and had an uneventful course for 1 year when he suddenly developed a submandibular abscess unrelated to his malignancy. He was admitted to a local hospital and surgically treated but died of sepsis 22 months after the MFH diagnosis.

\section{Discussion}

MFH of the spine is slow growing and might not manifest itself until nerve compression or a pathological fracture occurs. Bone destruction is characteristically not accompanied by periosteal reaction or new bone formation [4]. Treatment includes irradiation, embolization, and surgery with en-bloc resection being the only curative option $[4,6]$. It is known that MFH can be transplanted to the surgeon during operation [7] so special precautions are recommended. Chemotherapy is still controversial.

The mean life expectancy is 33 months after en-bloc resection and 15 months with palliative management [4].

\section{Conclusion}

We report a rare case of thoracic spine MFH with multiple bone metastases and spinal cord compression successfully managed with multidisciplinary therapy. The primary tumor shrunk significantly and did not grow again. The patient survived for 22 months with a good quality of life. To our knowledge, this is the longest survival reported for MFH of the spine presenting with spinal cord compression and treated palliatively. 


\section{Acknowledgement}

The authors express their gratitude to Dr. Hiroshi Nishihara for pathology evaluation, Dr. Akihiko Miyamoto for therapeutic irradiation, and Dr. Akimasa Nishio for preoperative embolization of the tumor.

\section{Statement of Ethics}

The study complied with guidelines for human studies. The subject gave informed consent for treatment and the use of images. The case report was deemed exempt by the institute's committee on human research.

\section{Disclosure Statement}

The authors declare that there are no conflicts of interest.

\section{Funding Sources}

None.

\section{Author Contributions}

Ivan Sekiguchi wrote and prepared the manuscript, and all the authors participated in the diagnosis and treatment. All authors have read, reviewed, and approved the case report.

\section{References}

1 O'Brien JE, Stout AP. Malignant fibrous xanthomas. Cancer. 1964;17:1445-55.

2 Kellett RJ, Dearnaley JN. Malignant fibrous histiocytoma with diffuse spinal nerve involvement. J Clin Pathol. 1976;29:910-5.

3 Sarcoma Meta-Analysis Collaboration. Adjuvant chemotherapy for localised resectable soft-tissue sarcoma of adults: meta-analysis of individual data. Sarcoma Meta-Analysis Collaboration. Lancet. 1997;350(9092): 1647-54.

4 Teng H, Xinghai Y, Wei H, Huang Q, Xiao J, Zhang C. Malignant fibrous histiocytoma of the spine: a series of 13 clinical case reports and review of 17 published cases. Spine (Phila Pa 1976). 2011 Oct;36(22):E1453-62.

5 Matushansky I, Charytonowicz E, Mills J, Siddiqi S, Hricik T, Cordon-Cardo C. MFH classification: differentiating undifferentiated pleomorphic sarcoma in the 21st century. Expert Rev Anticancer Ther. 2009 Aug;9(8):113544.

6 Somasundaram A, Wicks RT, Lata AL, Qasem SA, Hsu W. En bloc spondylectomy for primary malignant fibrous histiocytoma of the thoracic spine with aortic involvement: case report. J Neurosurg Spine. 2015 Apr;22(4): 399-405.

7 Gärtner HV, Seidl C, Luckenbach C, Schumm G, Seifried E, Ritter H, et al. Genetic analysis of a sarcoma accidently transplanted from a patient to a surgeon. N Engl J Med. 1996 Nov 14;335(20):1494-6. 